\title{
Overexpression of Notch1 is associated with the progression of cervical cancer
}

\author{
YAN SUN ${ }^{1}$, RUI ZHANG ${ }^{1}$, SHUJUAN ZHOU ${ }^{1}$ and YUQIANG JI ${ }^{2}$ \\ ${ }^{1}$ Department of Gynaecology and Obstetrics, General Hospital of Zaozhuang Mining Group, Zaozhuang, Shandong 277000; \\ ${ }^{2}$ Department of Cardiovascular Medicine, No. 1 Hospital of Xi'an City, Xi'an, Shaanxi 710002, P.R. China
}

Received July 17, 2014; Accepted March 25, 2015

DOI: $10.3892 / \mathrm{ol} .2015 .3143$

\begin{abstract}
Cervical cancer is the third most common malignancy worldwide, accounting for 250,000 mortalities annually. Notch1, an important regulator of cell-fate decisions and differentiation, has been found to be overexpressed in certain types of cancer. However, the role of Notch1 in cervical carcinogenesis remains unclear. In the present study, immunohistochemical staining and western blot analysis revealed that Notch1 expression was significantly higher in cervical cancer tissues than that in normal cervical tissues. Furthermore, statistical analysis revealed that Notch1 expression was significantly associated with tumor differentiation and tumor stage. These findings indicated that Notch1 expression was associated with the progression of cervical cancer. The western blot assay also identified a positive correlation between Notch1 and Ki67 expression in cervical cancer tissues, which suggested that Notch1 expression may be associated with the proliferation of cervical cancer. In order to further evaluate the specific role of Notch1 in cervical cancer progression, its expression in $\mathrm{SiHa}$ and C33A cells was knocked down using small interfering RNA. It was revealed that the knockdown of Notch1 in $\mathrm{SiHa}$ and C33A cells resulted in significant inhibition of cell proliferation and colony formation in vitro. These results indicated that Notch1 was able to promote cell proliferation in cervical cancer. In conclusion, the results of the present study indicated that Notch1 may function as a promoter in cervical carcinogenesis.
\end{abstract}

\section{Introduction}

Cervical cancer is one of the leading causes of cancer-associated mortalities among women worldwide, accounting for $>270,000$ mortalities annually $(1,2)$. Although certain advances have been achieved in the early detection and systemic treatment

Correspondence to: Dr Yuqiang Ji, Department of Cardiovascular Medicine, No. 1 Hospital of Xi'an City, 30 Fenxiang South Street, Xi'an, Shaanxi 710002, P.R. China

E-mail: jiyuqiang112299@126.com

Key words: cervical cancer, Notch1, immunohistochemical staining of patients receiving chemotherapy, hormonal therapy and immunotherapy, the precise mechanisms underlying cervical carcinogenesis have remained elusive. Previous studies have suggested that carcinogenesis is closely associated with the aberrant expression of certain proteins associated with cell signaling regulation $(3,4)$. These genetic and molecular events ultimately contribute to the initiation and progression of tumors. Therefore, the identification of alterations to specific genes associated with cervical cancer may provide a conceptual framework for the future analysis of this complex disease.

The Notch signaling pathway is an important regulator of embryonic cell fate decisions, proliferation and patterning (5). Notch receptors are large, transmembrane epidermal growth factor-like repeat-containing proteins that contain an extracellular domain responsible for ligand binding, and a cytoplasmic domain involved in signal transduction $(6,7)$. In total, mammals express four Notch receptors: Notch1, 2, 3 and 4, which are activated by Serrate (Jagged) and Delta (Delta-like) ligands expressed on the surface of adjacent cells. This activation results in a sequence of proteolytic cleavage events in the receptor, initially by a disintegrin and metalloproteinase enzyme and then by the $\gamma$-secretase complex, which releases the Notch intracellular domain (NICD) from the membrane (8). The NICD is subsequently translocated to the nucleus where it interacts with CSL transcription factors and activates the expression of target genes (9).

In addition to its central role in the developmental process, Notch1 has been reported to be deregulated in a variety of types of cancer (10). Hyperactivated Notch1 signaling has been implicated in the development of T-cell lymphoblastic leukemia (11), colon cancer (12) and breast cancer (13). In a further study, accumulative events revealed that Notch1 was a major factor in tumorigenesis due to its involvement in the regulation of cell growth, proliferation, differentiation and apoptosis (14). By contrast, Nicolas et al (15) reported that Notch1 was able to function as a tumor-suppressor gene in mammalian skin, which indicated that the role of Notch1 in tumorigenesis remains controversial. At present, the precise association between Notch1 and cervical cancer is yet to be elucidated. A better understanding of the role of Notch1 in the development of cervical cancer may provide novel insights into the process of tumorigenesis.

The present study used immunohistochemical staining to examine the expression of Notch1 in human cervical 
cancer tissues and normal cervical tissues. In addition, the association between Notch1 and various clinicopathological characteristics was investigated. Furthermore, the effect of Notch1 on the proliferation of cervical cancer cells was evaluated by small interfering (si)RNA-mediated Notch1 knockdown.

\section{Materials and methods}

Cell lines and cell culture. Human cervical cancer cell lines HeLa, SiHa, C33A, HT-3 and Caski were purchased from the American Type Culture Collection (Manassas, VA, USA). The HeLa, SiHa and C33A cells were maintained in Dulbecco's modified Eagle's medium (DMEM; Sigma-Aldrich, St. Louis, MO, USA) supplemented with $10 \%$ fetal bovine serum (FBS; Invitrogen Life Technologies, Carlsbad, CA, USA). The HT-3 cells were maintained in McCoy's 5A medium (Sigma-Aldrich) with 15\% FBS, and the Caski cells were maintained in RPMI-1640 medium (Invitrogen Life Technologies) with $10 \%$ FBS. All cell lines were incubated at $37^{\circ} \mathrm{C}$ in an atmosphere of $5 \% \mathrm{CO}_{2}$.

Immunohistochemical analysis. A total of 84 samples, including 59 cervical cancer tissues and 25 adjacent normal cervical tissues, were obtained via surgical resection from patients previously diagnosed with cervical cancer who had not received chemotherapy, immunotherapy or radiotherapy at the Zaozhuang Group Hospital (Zaozhuang, China) between July 2011 and June 2013. This study was approved by the ethics committee of the Zaozhuang Group Hospital, and patients provided informed consent prior to sample collection.

Immunohistochemistry ( $\mathrm{ICH}$ ) procedures were performed on $5-\mu \mathrm{m}$ sections prepared from $10 \%$ formalin (Luoyang Haohua Chemical Reagent Co., Ltd., Luoyang, China)-fixed, paraffin (Shanghai Hua Lingkang Complex Equipment Plant, Shanghai, China)-embedded tissues. Following deparaffinization with xylene (Tianjin Tianli Chemical Reagent Co. Ltd., Tianjin, China) and rehydration, sections were retrieved in $10 \mathrm{mM}$ citrate buffer (Tianjin Tianli Chemical Reagent Co., Ltd.) (pH 6.0) and blocked with $3 \% \mathrm{H}_{2} \mathrm{O}_{2}$ (Tianjin Tianli Chemical Reagent Co., Ltd.). The sections were then washed three times with phosphate-buffered saline (PBS; Tianjin Tianli Chemical Reagent Co., Ltd.) and incubated with primary polyclonal goat anti-human Notch1 (dilution, 1:100; cat. no. sc-6014; Santa Cruz Biotechnology, Inc., Dallas, TX, USA) antibody overnight at $4^{\circ} \mathrm{C}$. Next, the sections were incubated with horseradish peroxidase-conjugated secondary rabbit anti-goat IgG antibodies (dilution, 1:1,000; cat. no. ZB-2306; Beijing Zhongshan Golden Bridge Biotechnology Co., Ltd., Beijing, China) for $30 \mathrm{~min}$ at room temperature, visualized using diaminobenzidine (Beijing Zhongshan Golden Bridge Biotechnology Co., Ltd.) and counterstained with hematoxylin (Sigma-Aldrich).

The sections were observed under a light microscope (CX21; Olympus Corporation, Tokyo, Japan) and independently scored by two investigators. The final score was determined by multiplying the staining intensity (scored as: 1 , no staining; 2 , weak staining; and 3 , strong staining) by the percentage of positive cells (scored as: $0,0-10 \%$ positive cells; $1,10-25 \%$ positive cells; $2,26-50 \%$ positive cells; 3 , $51-75 \%$ positive cells; and 4, 76-100\% positive cells). Samples with a score of $>3$ were considered positive.

Western blot analysis. All tumor tissues and cells were lysed on ice with lysis buffer (50 mM Tris-HCl, $\mathrm{pH} 7.4$; $150 \mathrm{mM} \mathrm{NaCl} ; 2 \mathrm{mM}$ EDTA; $1 \% \mathrm{NP}-40$; and 0.1\% SDS; Sigma-Aldrich) containing a protease inhibitor (Complete Mini; Roche Diagnostics, Branchburg, NJ, USA). The lysates were then separated by $10 \%$ SDS-PAGE (Sigma-Aldrich) and transferred onto PVDF membranes (EMD Millipore, Billerica, MA, USA). Subsequent to blocking with $5 \%$ fat-free milk, the membranes were incubated with primary polyclonal goat anti-human Notch1 (dilution, 1:500; cat. no. sc-6014; Santa Cruz Biotechnology Inc.), polyclonal rabbit anti-human Ki67 (dilution, 1:500; cat. no. sc-15402; Santa Cruz Biotechnology, Inc.) and monoclonal mouse anti-human $\beta$-actin (dilution, 1:1,000; cat. no. sc-47778; Santa Cruz Biotechnology, Inc.) antibodies at $4^{\circ} \mathrm{C}$ overnight. This was followed by incubation with horseradish peroxidase-conjugated rabbit anti-goat (dilution, 1:5,000; ZB-2306; ZSGB-Bio Technology Co., Ltd, Beijing, China), goat anti-rabbit (dilution, 1:5,000; cat. no. A6124; Thermo Fisher Scientific, Waltham, MA, USA) or goat anti-mouse (dilution, 1:5,000; cat. no. A16090; Thermo Fisher Scientific) IgG secondary antibodies at room temperature for $1 \mathrm{~h}$. The proteins were detected by enhanced chemiluminescence (ECL; EMD Millipore) and visualized on X-ray film. The relative expression of Notch1 and Ki67 was determined by the Quantity One system (Bio-Rad Laboratories, Inc., Hercules, CA, USA), and normalized to $\beta$-actin for quantification. The intensity of protein expression was scored according to the ratio of Notch1/Ki67 to $\beta$-actin, respectively, as follows: Notch1, negative expression, $<0.25$; weak expression, 0.25-0.5; and strong expression, $>0.5$; and Ki67, negative expression, $<0.5$; weak expression, $0.5-1$; and strong expression, $>1$.

cDNA microarray database analysis. A cDNA microarray database (GSE5787) from an established human cervical cancer study was retrieved using GEO profiles (http:// www.ncbi.nlm.nih.gov./geoprofiles/?term=GSE5787) and Pearson's correlation was used to analyze the association between Notch1 and Ki67 mRNA expression.

RNA interference. A Notch1-targeting siRNA oligonucleotide was obtained from GenePharma Co., Ltd. (Shanghai, China). The sequences of the Notch1-targeting siRNA nucleotides were as follows: siNotch1-1, 5'-GATCCTGGCGGGAAGTGTGAAGCGT-3'; si Notch1-2, 5'-AGCTTAATGGCGGGAAGTGTGAAGC-3'; and siNotch1-3, 5'-AGACGCTTCACACTTCCCGCCATTA-3'. The negative control was designed as random sequence. A total of $1 \times 10^{5}$ cells/well were seeded into six-well plates and transfected with siRNA oligonucleotides using Lipofectamine ${ }^{\circledR} 2000$ (Invitrogen Life Technologies) according to the manufacturers' instructions.

Cell proliferation. The cells were seeded into six-well plates at a density of $5 \times 10^{4}$ cells/well and incubated in DMEM supplemented with $10 \% \mathrm{FBS}$ at $37^{\circ} \mathrm{C}$ in an atmosphere of $5 \%$ 
Table I. Correlation between Notch1 expression and clinicopathological characteristics.

\begin{tabular}{|c|c|c|c|c|}
\hline Characteristic & All cases & Notch1-negative ${ }^{a}$ cases & Notch1-positive ${ }^{\mathrm{b}}$ cases & P-value \\
\hline Total, $\mathrm{n}$ & 59 & 15 & 44 & \\
\hline Age, n (\%) & & & & 0.480 \\
\hline$\geq 55$ years & $23(39)$ & $7(47)$ & $16(36)$ & \\
\hline$<55$ years & $36(61)$ & $8(53)$ & $28(64)$ & \\
\hline Tumor differentiation, $\mathrm{n}(\%)$ & & & & $<0.01$ \\
\hline Well/moderate & $28(47)$ & $13(87)$ & $15(34)$ & \\
\hline Poor & $31(53)$ & $2(13)$ & $29(66)$ & \\
\hline Tumor stage, n (\%) & & & & 0.201 \\
\hline I-II & $35(59)$ & $11(73)$ & $24(55)$ & \\
\hline III-IV & $24(41)$ & $4(27)$ & $20(45)$ & \\
\hline Lymph node metastasis, n (\%) & & & & 0.714 \\
\hline Negative & $37(63)$ & $10(67)$ & $27(61)$ & \\
\hline Positive & $22(37)$ & $5(33)$ & $17(39)$ & \\
\hline
\end{tabular}

${ }^{\mathrm{a}}$ Score, $\leq 3$; ${ }^{\mathrm{b}}$ score, $>3 ;{ }^{\mathrm{c}} \chi^{2}$ test.

A

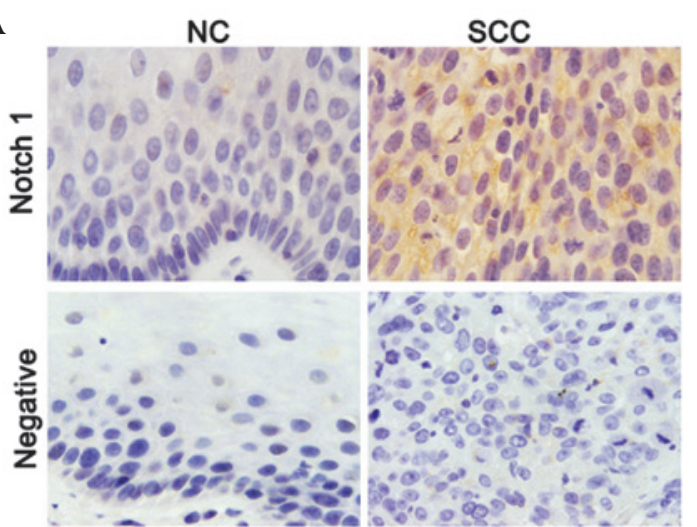

C

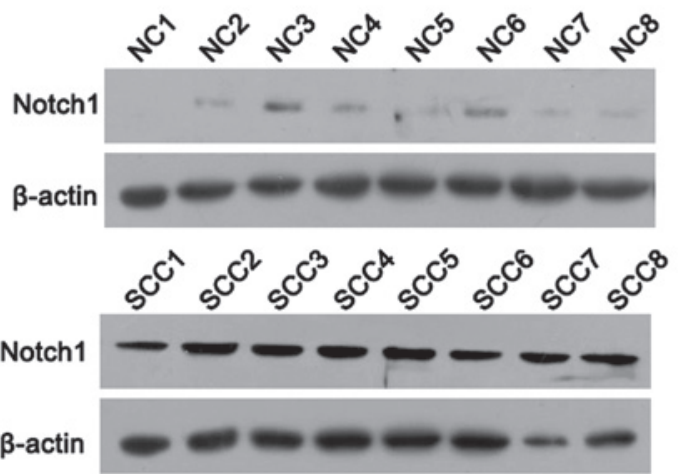

B

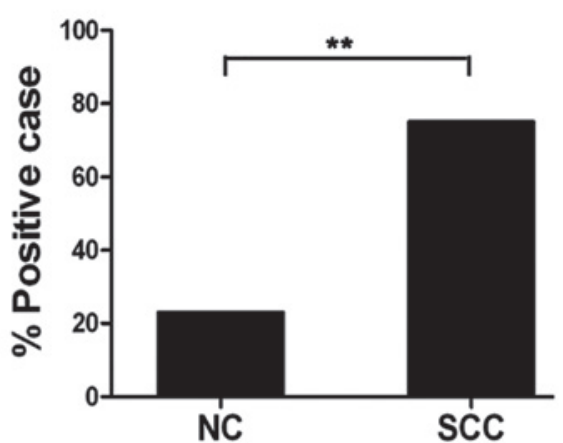

D

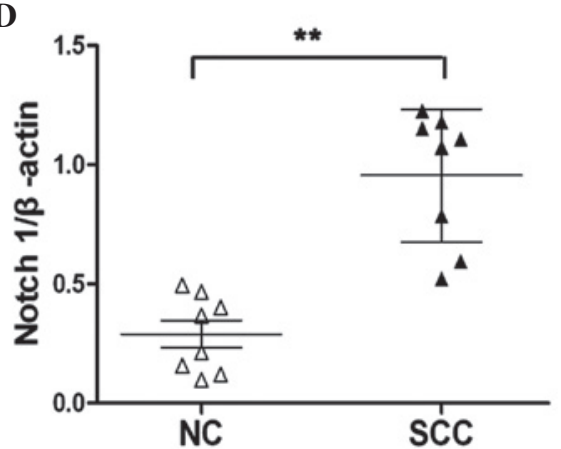

Figure 1. Expression of Notch1 is enhanced in cervical cancer tissues. (A) Immunohistochemical analysis of Notch1 expression in normal cervical tissues and cervical cancer tissues (magnification, x400). (B) The percentage of Notch1-positive expression in cervical cancer tissues was significantly higher than that of normal cervical tissues. (C) Western blotting identified the expression of Notch1 in cervical cancer and normal cervical tissues. (D) Quantitative analysis of Notch1 expression in normal cervical tissues and cervical cancer tissues normalized to $\beta$-actin expression. NC, normal cervical tissues; SCC, squamous cervical cancer. Results are expressed as the mean \pm standard deviation. ${ }^{* *} \mathrm{P}<0.01$ vs. control.

$\mathrm{CO}_{2}$. The cells were harvested and counted on days $1,3,5$, and 7 using a hemocy tometer (Shanghai Qiujing Biochemical Reagent Instrument Co., Ltd., Shanghai, China). Cell proliferation was then assessed following the construction of cell growth curves.
In order to analyze the cell viability, cells were seeded into 96-well plates at a density of $1 \times 10^{3}$ cells/well and cultured at $37^{\circ} \mathrm{C}$ for 7 days, as aforementioned. MTT assays were then performed according to standard protocols (16). In brief, $20 \mu 1$ MTT $(5 \mathrm{mg} / \mathrm{ml})$ was added to each well and incubated for $4 \mathrm{~h}$ at 


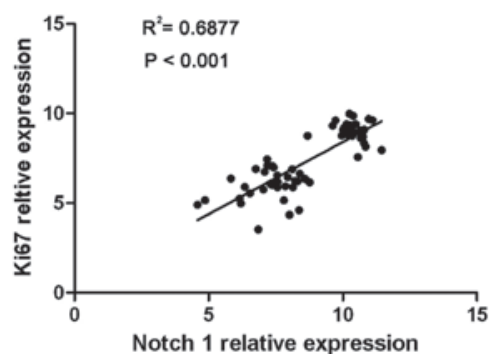

C

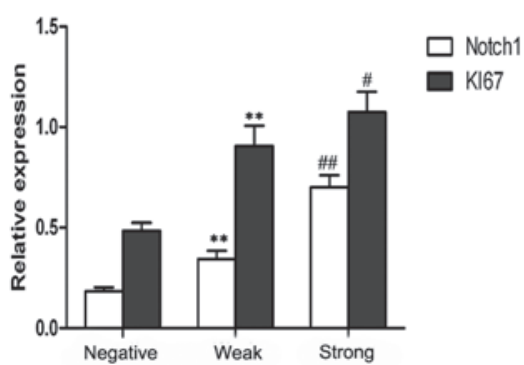

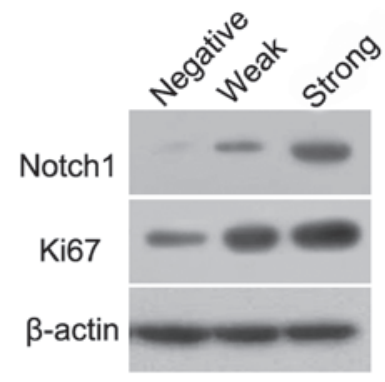

D

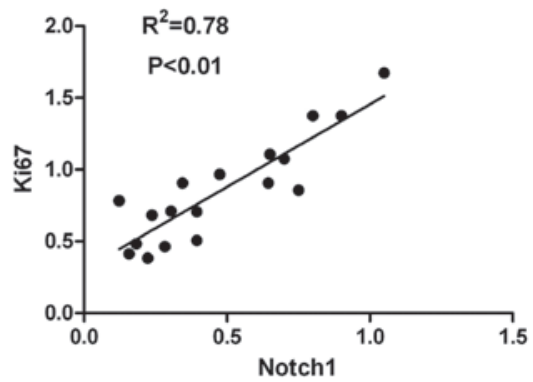

Figure 2. Notch1 and Ki67 expression are positively correlated in cervical cancer tissues. Correlation analysis between the expression of Notch1 and Ki67 in cervical cancer. (A) Correlations between Notch1 and Ki67 messenger RNA expression from microarray databases (GSE5787) of human cervical cancer were analyzed. (B) The expression of Notch1 and Ki67 in cervical cancer tissues was determined by western blotting. (C) Quantitative analysis of the relative expression of Notch1 and Ki67 in cervical cancer tissues based on the expression of the loading control, $\beta$-actin. ${ }^{* *} \mathrm{P}<0.01$ vs. negative expression. ${ }^{*} \mathrm{P}<0.05$ and ${ }^{\# \#} \mathrm{P}<0.01$ vs. weak expression. Six cases were included in each group. (D) Notch1 expression was positively correlated with Ki67 expression in human cervical cancer tissues.

A

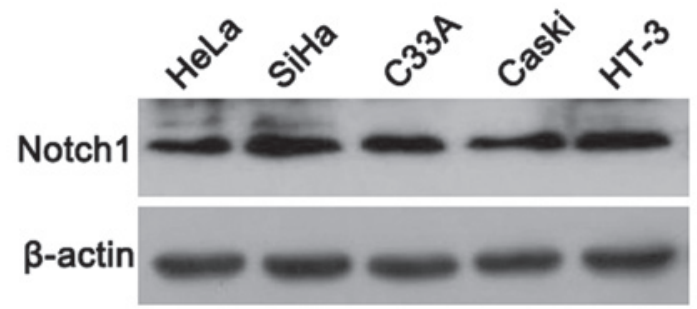

B

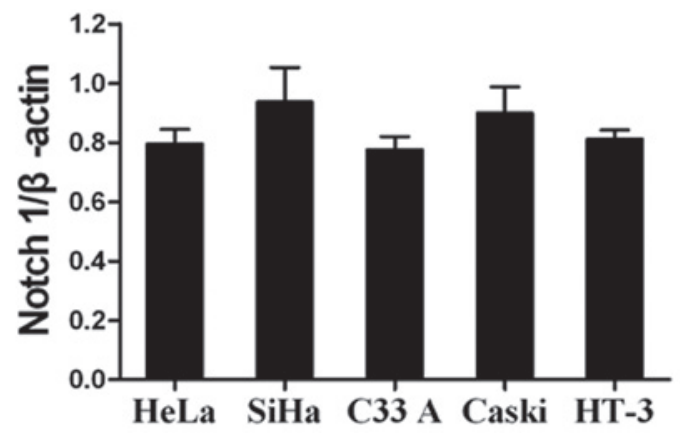

Figure 3. Expression of Notch1 is upregulated in cervical cancer cell lines. (A) The expression of Notch1 in HeLa, SiHa, C33A, Caski and HT-3 cells was determined by western blot analysis. (B) Relative expression of Notch1 in HeLa, SiHa, C33A, Caski and HT-3 cells was analyzed according to the Notch $1 / \beta$-actin ratio. $\beta$-actin was used as the loading control. The results are expressed as the mean \pm standard deviation from three experiments performed in duplicate.

$37^{\circ} \mathrm{C}$, followed by $150 \mu \mathrm{l}$ dimethyl sulfoxide (Sigma-Aldrich). The absorbance was read at $540 \mathrm{~nm}$ using a Bio-Rad 3350 microplate reader (Bio-Rad Laboratories, Inc.).
Colony formation assay. The cells were plated in $60-\mathrm{mm}$ culture dishes at a density of 300 cells/well and cultured in DMEM supplemented with $10 \% \mathrm{FBS}$ at $37^{\circ} \mathrm{C}$ for 3 weeks. Next, the colonies were rinsed with PBS, fixed with absolute methanol (Xi'an Chemical Reagent Factory, Xi'an, China) for 15 min and stained with Giemsa solution (Beijing DingGuo ChangSheng Biotechnology Co., Ltd., Beijing, China) for $30 \mathrm{~min}$. Colonies containing $\geq 50$ cells were considered to be positive.

Statistical analysis. Data are expressed as the mean \pm standard deviation. Statistical analysis was performed using SPSS software version 16.0 (SPSS Inc., Chicago, IL, USA). Differences between groups were analyzed using the $\chi^{2}$ test or Student's t-test, depending on the data type. Correlations between Notch1 and Ki67 expression were evaluated using the Pearson's correlation test. A value of $\mathrm{P}<0.05$ was considered to indicate a statistically significant difference.

\section{Results}

Notchl expression is enhanced in cervical cancer tissues. In order to determine the role of Notch1 in cervical carcinogenesis, its endogenous expression in human cervical cancer and normal cervical tissues was examined by immunohistochemical staining. As shown in Fig. 1A, the expression of Notch1 was primarily identified in the cytoplasm and membrane of cancer cells. Notch1 expression was significantly higher in cervical cancer tissues compared with that of normal cervical tissues $(\mathrm{P}<0.01)$. Notch1 protein was positively expressed in $23 \%(6 / 25)$ of the normal cervical tissue samples, and 
A

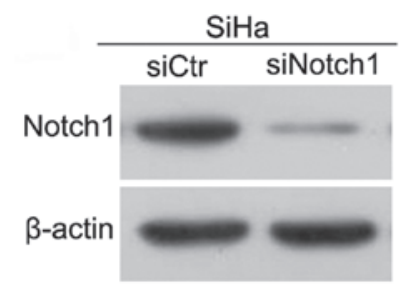

B

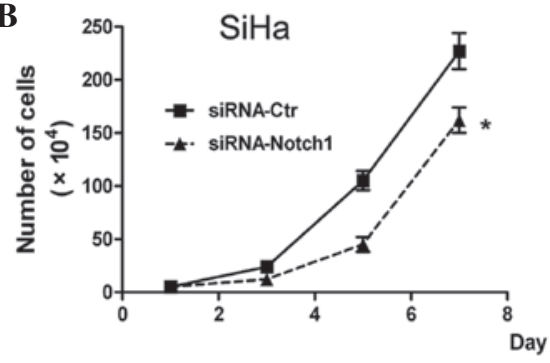

C

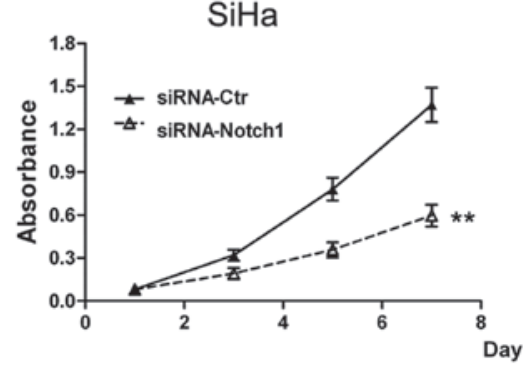

D

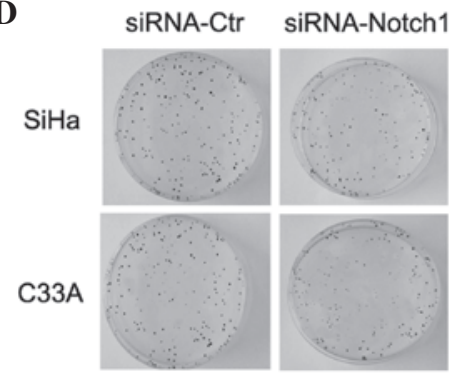

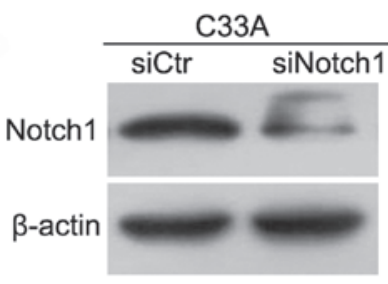

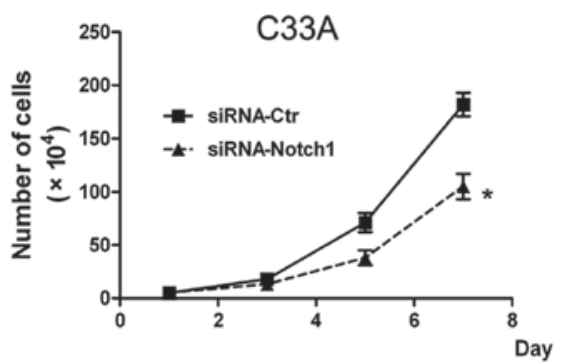

C33A
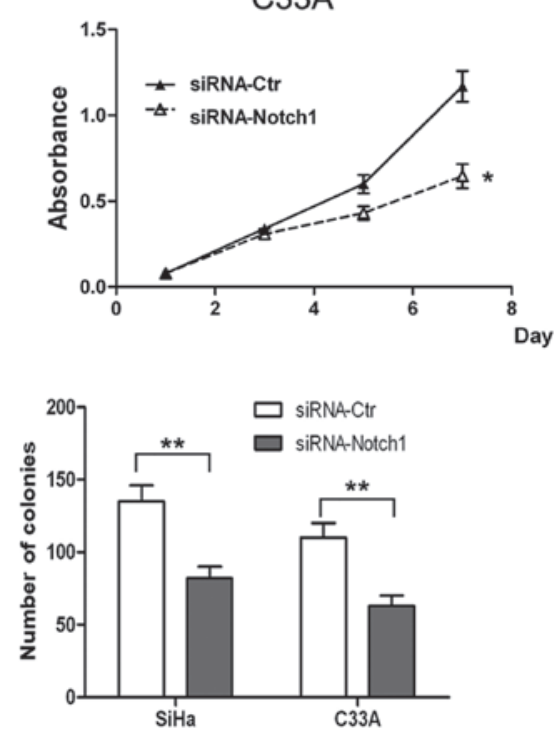

Figure 4. Knockdown of Notch1 inhibited the proliferation and colony formation of cervical cancer cells in vitro. SiHa and C33A cells were transfected with siRNA oligonucleotides and harvested following $24 \mathrm{~h}$ of incubation. (A) Western blotting revealed the knockdown of Notch1 in SiHa and C33A cells. (B) Knockdown of Notch1 inhibited the proliferation of SiHa and C33A cells in vitro. (C) Knockdown of Notch1 inhibited the viability of SiHa and C33A cells in vitro. (D) Knockdown of Notch1 inhibited the colony forming ability of SiHa and C33A cells. Results are presented as the mean \pm standard deviation from three experiments performed in duplicate. ${ }^{*} \mathrm{P}<0.05$ and ${ }^{* *} \mathrm{P}<0.01$ vs. control. siRNA, small interfering RNA; Ctr, control.

$75 \%(44 / 59)$ of the squamous cervical cancer tissue specimens (Fig. 1B). In order to further investigate the expression of Notch1 in cervical cancer, a western blot assay was performed using eight randomly selected normal cervical and cervical cancer tissues. In accordance with the IHC findings, it was revealed that the levels of Notch1 in cervical cancer tissues were significantly higher than those in normal cervical tissues $(\mathrm{P}<0.01)$. Together, these results indicated that Notch1 is highly expressed in cervical cancer, and is therefore likely to be associated with the progression of cervical cancer.

Notch1 expression is associated with tumor differentiation. Correlations between Notch1 expression and the clinicopathological characteristics of patients with cervical cancer were investigated. As shown in Table I, a significant positive correlation was observed between Notch1 expression and tumor differentiation $(\mathrm{P}<0.01)$, but not between Notch1 expression and patient age or lymph node metastasis status $(\mathrm{P}>0.05)$. The percentage of positive cases in the poorly-differentiated group was markedly higher than that in the well/moderately-differentiated group. This finding confirmed that Notch1 was closely associated with the progression of cervical cancer.

Notchl expression is positively correlated with Ki67 expression in cervical cancer tissues. Through analyzing a complementary (c)DNA microarray database (GSE5758) from an established human cervical cancer study, a significant, positive correlation was identified between Notch1 and Ki67 expression in cervical cancer (Fig. 2A). It is known that Ki67 is a marker of cell proliferation (17), therefore, these observations implied that Notch1 may also be involved in the proliferation of cervical cancer cells.

In order to determine whether increased Notch1 expression was associated with the proliferation of cervical cancer cells, 18 cervical cancer cases were randomly selected, and 
the expression of Notch1 and Ki67 was assessed by western blot analysis. A representative blot is shown in Fig. 2B, and the relative quantitative expression of Notch1 and Ki67 is summarized in Fig. 2C. Based on the intensity of protein expression, the cases were categorized into three grades; negative, weak and strong. It was established that the expression of Ki67 in cervical cancer was similar to that of Notch1 (Fig. 2C). Furthermore, the correlation analysis confirmed that Notch1 expression was significantly associated with Ki67 expression in cervical cancer $(\mathrm{r}=0.88 ; \mathrm{P}<0.01$; Fig. $2 \mathrm{~B})$. These data suggested that Notch1 was likely to be associated with the proliferation of cervical cancer cells.

Knockdown of Notchl inhibits the proliferation and colony formation of cervical cancer cells in vitro. The present study also analyzed the expression of Notch1 in the HeLa, SiHa, C33A, Caski and HT-3 cervical cancer cell lines by western blotting. The results revealed that Notch1 was highly expressed in all cervical cancer cell lines (Fig. 3A and B). This indicated that Notch signaling is likely to be activated in cervical cancer cell lines. In order to further investigate the specific role of Notch1 in the progression of cervical cancer, SiHa and C33A cells were randomly selected for use as an in vitro model for the assessment of Notch1 function in the subsequent experiments. First, the expression of Notch1 was knocked down in SiHa and C33A cells by siRNA. The western blot results of the knockdown are shown in Fig. 4A. It was revealed that Notch1 was significantly downregulated in the siRNA knockdown $\mathrm{SiHa}$ and C33A cells. Subsequently, a cell growth curve assay and MTT assay were performed. The cell growth curves demonstrated that Notch1 knockdown in SiHa and C33A cells resulted in a significant inhibition of cell proliferation $(\mathrm{P}<0.05$; Fig. $4 \mathrm{~B})$. In addition, the MTT assay analysis of $\mathrm{SiHa}$ and $\mathrm{C} 33 \mathrm{~A}$ cells revealed that Notch1 knockdown resulted in a significant decrease in cell viability (Fig. 4C). These findings confirmed the role of Notch1 in the promotion of cell proliferation.

A colony formation assay was also performed in order to determine whether Notch1 enhanced the proliferation ability of the cells. It was revealed that the number of formed colonies in Notch1 knockdown SiHa cells was significantly lower than that in the control cells $(\mathrm{P}<0.01$; Fig. 4D). Similar results were observed following Notch1 knockdown in C33A cells. Therefore, it was concluded that knockdown of Notch1 expression significantly inhibited the proliferation of $\mathrm{SiHa}$ and C33A cells in vitro. Previous studies have indicated that Notch1 regulates cell transformation and functions as an oncogene to promote tumor growth $(12,14)$. Collectively, the results of the present study indicated that Notch1 is a critical regulator of cervical cancer cell proliferation in vitro.

\section{Discussion}

Cervical cancer is the third most common type of malignant tumor and the fourth leading cause of cancer-associated mortalities among women worldwide (18). Particularly in certain developing countries, the incidence and mortality rates of cervical cancer remain high due to a lack of screening and appropriate therapeutic facilities and drugs (19). Increasing evidence has revealed that aberrant cell proliferation is associated with the dysregulation of signaling pathways that link co-regulated genes, which are required for cell homeostasis during malignant transformation (20). The canonical Notch1 signaling pathway is believed to be a fundamental transduction pathway involved in directly transmitting signals from the cell surface to the nucleus (21). Notch1 has been demonstrated to have essential roles in the regulation of tumor growth, invasion, metastasis and angiogenesis (11,22-24). Furthermore, the overexpression of Notch1 has been observed in numerous types of human cancer $(25,26)$, and Notch signaling has been reported to have an oncogenic role in breast (27), colorectal (28), ovarian (29), pancreatic and prostate cancer (30), as well as leukemia and lymphoma (31). However, the function of Notch1 in cervical cancer progression remains poorly understood.

In the present study, immunohistochemical staining and western blotting revealed that Notch1 expression was significantly higher in cervical cancer tissues than that in normal cervical tissues. This finding revealed a potential role for Notch1 in cervical carcinogenesis. However, the expression of Notch1 in cervical cancer remains controversial. Zagouras et al (32) reported that the expression of Notch1 in situ and in invasive squamous cancers of the cervix was higher than in normal cervical tissues (32). However, Talora et al (33) reported that the expression of Notch1 was highly cell- and context-specific. The present study found that elevated Notch1 expression was significantly associated with tumor differentiation, but not with age and lymph node metastasis status, which suggested that Notch signaling may be involved in the progression of cervical cancer. Taken together, these results indicate that Notch1 has an essential role in the development and progression of cervical cancer.

It has previously been reported that the Notch signaling pathway has a fundamental role in modulating the balance between cell proliferation, differentiation and apoptosis (5), and that continuous activated Notch1 signaling promotes cell proliferation (34) and survival (35).

In the present study, the reanalysis of cervical cancer cDNA from a microarray dataset (GSE5787) indicated that Ki67 expression was significantly associated with Notch1 expression in cervical cancer. Further investigation revealed that the expression of Ki67 was positively correlated with the expression of Notch1 in cervical cancer. Ki67 is a marker of cell proliferation. Accordingly, the results of the present study suggested that Notch1 expression was associated with cell proliferation, and that cervical cancer cells with high Notch1 expression may have a higher proliferative activity. As cell proliferation is regulated by multiple extracellular signals (36), the role of Notch1 in cervical carcinogenesis has remained elusive. In order to determine the precise role of Notch1 in tumor progression, its expression in the SiHa and C33A cervical cancer cell lines was knocked down by siRNA transfection. The cell growth curves and MTT assay revealed that the knockdown of Notch1 significantly inhibited the proliferation of cervical cancer cells in vitro. In addition, an inhibition of colony formation induced by the knockdown of Notch1 was observed in $\mathrm{SiHa}$ and C33A cells. These findings supported the hypothesis that activated Notch1 signaling promotes cervical cancer progression. These results are consistent with those of a previous study, which demonstrated that Notch1 had a pro-oncogenic role in the progression of cervical cancer (37). 
In conclusion, the present study demonstrated that Notch1 was highly expressed in cervical cancer, and that the increased expression of Notch1 promoted the progression of cervical cancer through enhancing cell proliferation. Collectively, these results suggested that Notch1 has an important role in cervical carcinogenesis, and is therefore a candidate therapeutic target for the treatment of cervical cancer. However, the function of Notch1 in regulating cancer progression is complex, and further studies are required in order to elucidate the mechanisms underlying the role of Notch1 in promoting the progression of cervical cancer.

\section{References}

1. Jemal A, Bray F, Center MM, Ferlay J, Ward E and Forman D: Global cancer statistics. CA Cancer J Clin 61: 69-90, 2011.

2. Saavedra KP, Brebi PM and Roa JC: Epigenetic alterations in preneoplastic and neoplastic lesions of the cervix. Clin Epigenetics 4: 13, 2012.

3. Chan LH, Wang W, Yeung W, Deng Y, Yuan P and Mak KK: Hedgehog signaling induces osteosarcoma development through Yap1 and H19 overexpression. Oncogene 33: 4857-4866, 2014.

4. Burgess AW, Faux MC, Layton MJ and Ramsay RG: Wnt signaling and colon tumorigenesis - a view from the periphery. Exp Cell Res 317: 2748-2758, 2011.

5. Artavanis-Tsakonas S, Rand MD and Lake RJ: Notch signaling: Cell fate control and signal integration in development Science 284: 770-776, 1999.

6. Egan S, St-Pierre B and Leow C: Notch receptors, partners and regulators: From conserved domains to powerful functions. In: Protein Modules in Signal Transduction. Pawson AJ (ed). Springer, New York, NY, pp273-324, 1998.

7. Callahan R and Egan SE: Notch signaling in mammary development and oncogenesis. J Mammary Gland Biol Neoplasia 9: $145-163,2004$.

8. Kopan R: Notch: A membrane-bound transcription factor. J Cell Sci 115: 1095-1097, 2002

9. Lai EC: Keeping a good pathway down: Transcriptional repression of Notch pathway target genes by CSL proteins. EMBO Rep 3: 840-845, 2002.

10. Harrison H, Farnie G, Brennan KR and Clarke RB: Breast cancer stem cells: Something out of notching? Cancer Res 70: 8973-8976, 2010.

11. Palomero T, Lim WK, Odom DT, et al: NOTCH1 directly regulates c-MYC and activates a feed-forward-loop transcriptional network promoting leukemic cell growth. Proc Natl Acad Sci USA 103: 18261-18266, 2006.

12. Zhang Y, Li B, Ji ZZ and Zheng PS: Notch1 regulates the growth of human colon cancers. Cancer 116: 5207-5218, 2010.

13. Bolós V, Mira E, Martinez-Poveda B, et al: Notch activation stimulates migration of breast cancer cells and promotes tumor growth. Breast Cancer Res 15: R54, 2013.

14. Jundt F, Anagnostopoulos I, Förster R, Mathas S, Stein H and Dörken B: Activated Notch1 signaling promotes tumor cell proliferation and survival in Hodgkin and anaplastic large cell lymphoma. Blood 99: 3398-3403, 2002.

15. Nicolas M, Wolfer A, Raj K, et al: Notch1 functions as a tumor suppressor in mouse skin. Nat Genet 33: 416-421, 2003.

16. Prasad R, Vaid M and Katiyar SK: Grape proanthocyanidin inhibit pancreatic cancer cell growth in vitro and in vivo through induction of apoptosis and by targeting the PI3K/Akt pathway. PLoS One 7: e43064, 2012

17. Jaks V, Barker N, Kasper M, et al: Lgr5 marks cycling, yet long-lived, hair follicle stem cells. Nat Genet 40: 1291-1299, 2008.
18. Ferlay J, Shin HR, Bray F, Forman D, Mathers C and Parkin DM: Estimates of worldwide burden of cancer in 2008: GLOBOCAN 2008. Int J Cancer 127: 2893-2917, 2010.

19. Mathew A and George PS: Trends in incidence and mortality rates of squamous cell carcinoma and adenocarcinoma of cervix - worldwide. Asian Pac J Cancer Prev 10: 645-650, 2009.

20. Hahn WC and Weinberg RA: Modelling the molecular circuitry of cancer. Nat Rev Cancer 2: 331-341, 2002.

21. Blanpain C, Lowry WE, Pasolli HA and Fuchs E: Canonical notch signaling functions as a commitment switch in the epidermal lineage. Genes Dev 20: 3022-3035, 2006.

22. Kunnumakkara AB, Anand P and Aggarwal BB: Curcumin inhibits proliferation, invasion, angiogenesis and metastasis of different cancers through interaction with multiple cell signaling proteins. Cancer Lett 269: 199-225, 2008.

23. Liu C, Li Z, Bi L, et al: NOTCH1 signaling promotes chemoresistance via regulating $\mathrm{ABCC} 1$ expression in prostate cancer stem cells. Mol Cell Biochem 393: 265-270, 2014.

24. Timmerman LA, Grego-Bessa J, Raya A, et al: Notch promotes epithelial-mesenchymal transition during cardiac development and oncogenic transformation. Genes Dev 18: 99-115, 2004.

25. Li D, Dong P, Wu C, Cao P and Zhou L: Notch1 overexpression associates with poor prognosis in human laryngeal squamous cell carcinoma. Ann Otol Rhinol Laryngol 123: 705-710, 2014.

26. Wu WR, Zhang R, Shi XD, et al: Notch1 is overexpressed in human intrahepatic cholangiocarcinoma and is associated with its proliferation, invasiveness and sensitivity to 5-fluorouracil in vitro. Oncol Rep 31: 2515-2524, 2014.

27. Reedijk M, Odorcic S, Chang L, et al: High-level coexpression of JAG1 and NOTCH1 is observed in human breast cancer and is associated with poor overall survival. Cancer Res 65: 8530-8537, 2005.

28. Bolós V, Blanco M, Medina V, Aparicio G, Díaz-Prado S and Grande E: Notch signalling in cancer stem cells. Clin Transl Oncol 11: 11-19, 2009.

29. Kunnimalaiyaan $\mathbf{M}$ and Chen H: Tumor suppressor role of Notch-1 signaling in neuroendocrine tumors. Oncologist 12: 535-542, 2007

30. Wang Z, Li Y, Banerjee S, et al: Down-regulation of Notch-1 and Jagged-1 inhibits prostate cancer cell growth, migration and invasion and induces apoptosis via inactivation of Akt, mTOR and NF- $\mathrm{BB}$ signaling pathways. J Cell Biochem 109: 726-736, 2010

31. Weng AP, Millholland JM, Yashiro-Ohtani Y, et al: c-Myc is an important direct target of Notch1 in T-cell acute lymphoblastic leukemia/lymphoma. Genes Dev 20: 2096-2109, 2006.

32. Zagouras P, Stifani S, Blaumueller CM, Carcangiu ML and Artavanis-Tsakonas S: Alterations in Notch signaling in neoplastic lesions of the human cervix. Proc Natl Acad Sci USA 92: 6414-6418, 1995.

33. Talora C, Sgroi DC, Crum CP and Dotto GP: Specific down-modulation of Notch1 signaling in cervical cancer cells is required for sustained HPV-E6/E7 expression and late steps of malignant transformation. Genes Dev 16: 2252-2263, 2002.

34. Go MJ, Eastman DS and Artavanis-Tsakonas S: Cell proliferation control by Notch signaling in Drosophila development. Development 125: 2031-2040, 1998.

35. Perumalsamy LR, Nagala M and Sarin A: Notch-activated signaling cascade interacts with mitochondrial remodeling proteins to regulate cell survival. Proc Natl Acad Sci USA 107: 6882-6887, 2010.

36. Yu CY, Liang GB, Du P and Liu YH: Lgr4 promotes glioma cell proliferation through activation of Wnt signaling. Asian Pac J Cancer Prev 14: 4907-4911, 2013.

37. Srivastava S, Ramdass B, Nagarajan S, Rehman M, Mukherjee G and Krishna S: Notch1 regulates the functional contribution of RhoC to cervical carcinoma progression. Br J Cancer 102: 196-205, 2010. 